Article

\title{
Analysis of Vibration Frequency of Carbon Nanotubes used as Nano-Force Sensors Considering Clamped Boundary Condition
}

\author{
Toshiaki Natsuki ${ }^{1,2, *}$ and Kairi Urakami ${ }^{3}$ \\ 1 Faculty of Textile Science and Technology, Shinshu University, 3-15-1 Tokida, Ueda-shi 386-8567, Japan \\ 2 Research Initiative for Supra-Materials, Shinshu University, 4-17-1 Wakasato, Nagano 380-8553, Japan \\ 3 Graduate School of Science and Technology, Shinshu University, 3-15-1 Tokida, Ueda-shi 386-8567, Japan; \\ 19fs303c@shinshu-u.ac.jp \\ * Correspondence: natsuki@shinshu-u.ac.jp; Tel.: +81-268-21-5421
}

Received: 3 September 2019; Accepted: 23 September 2019; Published: 24 September 2019

check for updates

\begin{abstract}
Carbon nanotubes (CNTs) can be used as atomic force microscope (AFM) probes since they are ideal tip materials with a small diameter, high aspect ratio, and stiffness. In this study, a model of CNTs clamped in an elastic medium is proposed as nanoscale force sensing AFM probes. The relationship between vibration frequency and axial force of the CNT probe clamped in an elastic medium is analyzed based on the Euler-Bernoulli beam model and the Whitney-Riley model. The clamped length of CNTs, and the elastic modulus of elastic medium affect largely on the vibration and the buckling stability of a CNT AFM probe. The result showed that the sensitivity to vibration increases as the applied loads increase. The critical load in which the vibration frequency decreases rapidly, moving to large ones with decreasing ratio of length to diameter of CNTs. The theoretical investigation on the vibration frequency of CNT loaded in the axial direction would give a useful reference for designing a CNT used as a nano-force sensor.
\end{abstract}

Keywords: carbon nanotubes; vibration frequency; nano sensor; modeling; simulation

\section{Introduction}

The atomic force microscope (AFM) has attracted great interest in many scientific and technological fields, including measuring the force or potential energy between a small tip and a sample. Since pioneering work by Iijima in 1991 [1], carbon nanotubes (CNTs) have been the subject of numerous studies, such as one of the most promising nano-sensor materials [2-6]. The applications of CNTs used as different sensors [6-8] have been broad due to their nanoscale dimensions and unique properties. A combination of the superb mechanical and physical properties, chemical stability, and high aspect ratio of CNTs make them ideal materials for use as scanning probe microscope tips, especially in atomic force microscope (AFM) [3,5,9-12]. The AFM has been considered a powerful tool for morphology analysis of nanoscale materials, and nano-structures such as quantum dots (QDs). Most of the AFM probes are made out of silicon or silicon nitride, by the micromachining and electron beam deposition techniques. At present, commercially available silicon probe tips commonly have a radius of curvature of approximately 5-15 $\mathrm{nm}$ [13]. The poorly characterized silicon and nitride probe tips currently employed in AFM, however, limited some applications since these conventional silicon tips could easily break during an impact on the scanned surface. The resolution of AFM imaging for surface structure analysis mainly depends on the tip diameter of CNTs and their aspect ratio [14]. Therefore, the CNTs are potentially ideal materials to serve as components in an AFM probe and sensor because of their robust mechanical properties and well-defined geometry with small diameter and high aspect ratio. 
Lieber's group proposed the potential application of the CNT used as AFM tips ten years ago [10,15]. The investigation showed that individual single-walled and multi-walled CNT tips exhibited much better lateral resolution than that of the Si tips in the tapping mode AFM measurement [16].

It is quite difficult to control experimentally individual CNTs due to an extremely small size. However, chemical vapor deposition (CVD) technology allowed CNT growth, easily and directly on desired substrates. A variety of forms, including controlling the diameter and the length of CNTs, can be achieved since the advanced CVD technology was developed [17-19]. To investigate the potential applications of CNTs as AFM tip and nano-force sensor, some experimental and theoretical studies have been reported and studied on the mechanical stability of CNTs [20-27].

Using the direct growth of CNT on silicon-cantilever-tip, commercially available silicon AFM tips of CNT can be fabricated by CVD. The grown CVD tips were so long that the molecular-scale CVD nanotube probes could be used to image isolated biological macromolecules at high resolution [28]. The measurement accuracy of the image contrast depends on not only the stiffness of the CNT tips but also their geometry. The CNT tips can retain and recover perfect structure of CNT attached to a hard surface due to higher mechanical properties of CNT, which is not as brittle as silicon. The stability of CNT tips subjected to compressive loading was investigated based on the continuum mechanics method, and the molecule dynamics. Based on the molecular dynamics (MD) simulation, Yakobson et al. firstly investigated and discussed different instability patterns of CNTs under compressive load [29]. The simulation results showed that CNTs were remarkably resilient in sustaining extreme strain without sings of brittleness. Using continuum mechanics and analytical techniques, the effects of the parameters, such as the dimension and clamping boundary conditions on the buckling stability and the vibration property of CNTs were analyzed and discussed [30,31]. The continuum model and approximations allowed us to obtain formulations relatively simply and were less computationally expensive than atomistic approaches. In the report [32], the dynamic properties of nano-resonators are relevant in the context of multifrequency AFM. They reviewed the development of multifrequency force microscopy methods, highlighting the five most prominent approaches.

Some theoretical approaches were employed to predict and analyze the mechanical properties of CNT structures. These analytical theories and methods included with the nonlocal continuum mechanics, in which stress field at a reference position depends not only on the strain at that position but also on strains at all other points in the domain [33-36]. Barretta et al. presented a stress-driven two-phases constitutive mixture by a convex combination of local and nonlocal phases [37]. The local-nonlocal mixture was denoted as mixture Eringen integral model and the local elastic fraction of the mixture had a beneficial effect. A contribution in the framework of non-local constitutive models for non-homogeneous elastic materials was addressed by de Sciarra [38]. He also presented a thermodynamically consistent formulation of the nonlocal plasticity in the framework of the internal variable theory of inelastic behavior $[39,40]$.

In this work, we proposed that CNTs could be used as nanoscale force sensing AFM probes because the vibration frequency of CNTs is very sensitive to small loading values. The buckling behavior significantly affects the performance of CNTs used as structural elements of sensor materials. Thus, more efforts should focus on studying their buckling and vibration characteristics. In the work, a cantilever beam model of CNTs clamped on an elastic substrate was proposed and used as a nano-force sensor, and the relationship between the vibration frequency and the axial loading are analyzed and discussed based on Euler-Bernoulli model and the Whitney-Riley model.

\section{Modeling Procedure}

\subsection{Fundamental Equations}

Figure 1 shows an analytical model of the CNT probe clamped in an elastic medium. As shown in Figure $1 b, L$ and $D$ are the length and the diameter of the CNT probe, and $L_{1}$ and $L_{2}$ present the clamped and exposed lengths, respectively. $k_{w}$ is a spring constant relative to the elastic medium, thus, 
the interaction force, $p$, between the CNT and the surrounding elastic medium can be described by the spring constant $\left(k_{w}\right)$ using Whitney-Riley model [41], given by

$$
p=k_{w} w
$$

where $w$ is the vibrational deflection of the CNTs.

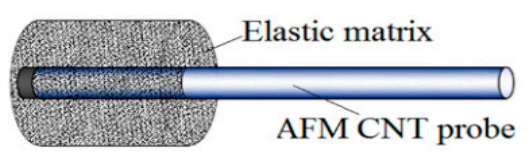

(a)

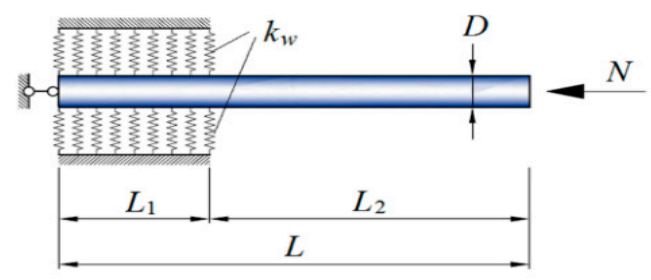

(b)

Figure 1. (a) A proposed atomic force microscope (AFM) carbon nanotube (CNT) probe clamped in an elastic medium, and $(\mathbf{b})$ the analytical modeling of the clamped CNT.

The governing differential equation of the flexural vibration for a CNT under an axial compressive loading $N$ based on the Euler-Bernoulli beam model, is expressed in [22]

$$
E I \frac{\partial^{4} w}{\partial x^{4}}+N \frac{\partial^{2} w}{\partial x^{2}}+\rho A \frac{\partial^{2} w}{\partial t^{2}}=p
$$

where $x$ and $t$ are the longitudinal coordinate and time, respectively. $E$ is the elastic modulus, and $I$ is the moment of inertia. $\rho$ and $A$ are the cross-section of CNT, respectively. $p$ is the distributed transverse pressure acting on the CNT per unit axial length, described as Equation (1). In the motion equation, the shear force stresses were negligible due to the assumption that the cross section remained normal to then neutral axis after deformation.

Based on the Euler-Bernoulli beam equation in Equation (2), the governing differential equations governing the motion of CNTs are described by the following two equations:

$$
\begin{array}{cc}
E I \frac{\partial^{4} w_{1}}{\partial x^{4}}+N \frac{\partial^{2} w_{1}}{\partial x^{2}}+\rho A \frac{\partial^{2} w_{1}}{\partial t^{2}}=-k_{w} w_{1}, & 0 \leq x \leq L_{1} \\
E I \frac{\partial^{4} w_{2}}{\partial x^{4}}+N \frac{\partial^{2} w_{2}}{\partial x^{2}}+\rho A \frac{\partial^{2} w_{2}}{\partial t^{2}}=0, & L_{1} \leq x \leq L
\end{array}
$$

where $w_{j}(j=1,2)$ are the vibrational deflections of the clamped and exposed parts of the CNTs, respectively.

The deflection of the CNT has different vibration amplitudes $Y_{j}(x), j=1,2$ for the clamped and exposed parts, the displacements of the vibration solution are given as

$$
w_{j}=Y_{j}(x) e^{i \omega t}, \quad j=1,2
$$

where $\omega$ is the vibrational frequency of the CNT. 
Substituting Equation (5) into Equations (3) and (4), the governing equations of the vibration property for CNT are obtained as

$$
\begin{gathered}
Y_{1}^{(4)}+2 \alpha Y_{1}^{\prime \prime}+\beta=0,0 \leq x \leq L_{1}, \\
Y_{2}^{(4)}+2 \alpha Y_{2}^{\prime \prime}-\gamma=0, \quad L_{1} \leq x \leq L,
\end{gathered}
$$

where

$$
\alpha=\frac{N}{2 E I}, \quad \beta=\frac{k_{w}-\rho A \omega^{2}}{E I}, \quad \gamma=\frac{\rho A \omega^{2}}{E I}
$$

\subsection{Solution of Governing Differential Equations}

By solving the fourth-order polynomial function in Equations (6) and (7), the solutions of the governing differential equations can be given as follows:

(1) If $\omega^{2} \leq \frac{1}{\rho A}\left(k_{w}-\frac{N^{2}}{4 E I}\right)$, we obtain

$$
Y_{1}(x)=e^{\lambda x}\left(A_{1} \cos \phi x+A_{2} \sin \phi x\right)+e^{-\lambda x}\left(A_{3} \cos \phi x+A_{4} \sin \phi x\right)
$$

where

$$
\lambda=\frac{1}{\sqrt{2}} \sqrt{p_{1}+\sqrt{p_{1}^{2}+p_{2}^{2}}}, \quad \phi=\frac{1}{\sqrt{2}} \sqrt{-p_{1}+\sqrt{p_{1}^{2}+p_{2}^{2}}}
$$

and

$$
p_{1}=-\alpha, \quad p_{2}=\beta-\alpha^{2}
$$

where $A_{j}(j=1,2, \cdots, 8)$ are arbitrary integration constants, which should be determined via boundary conditions.

(2) If $\frac{1}{\rho A}\left(k_{w}-\frac{N^{2}}{4 E I}\right)<\omega^{2}<\frac{k_{w}}{\rho A}$, we have

$$
Y_{1}(x)=A_{1} \cos p_{1} x+A_{2} \sin p_{1} x+A_{3} \cos p_{2} x+A_{4} \sin p_{2} x
$$

where

$$
P_{1}=\sqrt{\alpha-\sqrt{\alpha^{2}-\beta}}, \quad P_{2}=\sqrt{\alpha+\sqrt{\alpha^{2}-\beta}}
$$

(3) when $\omega^{2} \geq \frac{k_{w}}{\rho A}$

$$
Y_{1}(x)=A_{1} \cosh \eta x+A_{2} \sinh \mu x+A_{3} \cos \theta x+A_{4} \sin \theta x
$$

where

$$
\eta=\sqrt{-\alpha+\sqrt{\alpha^{2}-\beta}}, \quad \theta=\sqrt{\alpha+\sqrt{\alpha^{2}-\beta}}
$$

For the exposed part of CNT, the solution of Equation (7) gives

$$
Y_{2}(x)=e^{\varsigma x}\left(A_{5} \cos \omega x+A_{6} \sin \omega x\right)+e^{-\zeta x}\left(A_{7} \cos \omega x+A_{8} \sin \omega x\right)
$$

where

$$
\zeta=\sqrt{-\alpha+\sqrt{\alpha+\gamma^{2}}}, \quad \omega=\sqrt{\alpha+\sqrt{\alpha+\gamma^{2}}}
$$

Considering that the CNT probe clamped length $L_{1}$ in an elastic medium and exposed length $L_{2}$, the corresponding boundary conditions are given as follows: 
(1) at $x=0$, for clamped part

$$
\left.\frac{\partial^{2} w_{1}(x, t)}{\partial x^{2}}\right|_{x=0}=0,\left.\quad \frac{\partial^{3} w_{1}(x, t)}{\partial x^{3}}\right|_{x=0}+\left.2 \alpha \frac{\partial w_{1}(x, t)}{\partial x}\right|_{x=0}=0
$$

(2) at $x=L$, for exposed part subjected to compressive loading

$$
\left.\frac{\partial^{2} w_{2}(x, t)}{\partial x^{2}}\right|_{x=L}=0,\left.\quad \frac{\partial^{3} w_{2}(x, t)}{\partial x^{3}}\right|_{x=L}+\left.2 \alpha \frac{\partial w_{2}(x, t)}{\partial x}\right|_{x=L}=0
$$

(3) at the position $x_{1}=L_{1}$, which is the connecting position between clamped and exposed parts, the continuous conditions of the vibrational deflection are:

$$
\begin{gathered}
\left.w_{1}(x, t)\right|_{x=L_{1}}=\left.w_{2}(x, t)\right|_{x=L_{1}} \\
\left.\frac{\partial w_{1}(x, t)}{\partial x}\right|_{x=L_{1}}=\left.\frac{\partial w_{2}(x, t)}{\partial x}\right|_{x=L_{1}},\left.\frac{\partial^{2} w_{1}(x, t)}{\partial x^{2}}\right|_{x=L_{1}}=\left.\frac{\partial^{2} w_{2}(x, t)}{\partial x^{2}}\right|_{x=L_{1}} \\
\left.\frac{\partial^{3} w_{1}(x, t)}{\partial x^{3}}\right|_{x=L_{1}}+\left.2 \alpha \frac{\partial w_{1}(x, t)}{\partial x}\right|_{x=L_{1}}=\left.\frac{\partial^{3} w_{2}(x, t)}{\partial x^{3}}\right|_{x=L_{1}}+\left.2 \alpha \frac{\partial w_{2}(x, t)}{\partial x}\right|_{x=L_{1}}
\end{gathered}
$$

Substituting the vibrational deflections of the clamped and exposed parts of CNTs into the above given boundary conditions of Equations (20)-(22), we obtain the simultaneous equation, given in matrix form:

$$
\Omega\left[N, L_{1}, L_{2}\right]_{8 \times 8}\left[\begin{array}{c}
A_{1} \\
A_{2} \\
\vdots \\
A_{8}
\end{array}\right]=0
$$

where $\Omega\left[N, L_{1}, L_{2}\right]_{8 \times 8}$ is a $8 \times 8$ matrix concluding with parameters, such as the axial compressive load $N$, and lengths $L_{1}$ and $L_{2}$ of the clamped and exposed parts. The relationship between the vibration frequency and the compression load can be obtained from the eigenvalue $|\Omega|_{8 \times 8}=0$, corresponding to the nontrivial solution in Equation (23).

\section{Simulation Results and Discussion}

The vibrational properties of the CNTs were investigated based on the proposed analytical model. When the axial load is applied to the CNTs, its vibration frequency will change with the loading values. The change in the vibration frequency of CNTs with the compression load can be used as a nano-loading sensor. In this simulation, the diameter and the length of a CNT were $2 \mathrm{~nm}$ and $40 \mathrm{~nm}$, respectively. The layer thickness of the CNT was $0.34 \mathrm{~nm}$, the spacing of graphite. It was important to note that the presence of CNT defects could affect the vibration frequency of CNTs. However, the CNT defect caused the change of the elastic modulus of CNT. We can give the suitable elastic modulus to consider the CNT defect and to simulate the vibration frequency of CNT. Here, the material constants of CNTs were taken as: The elastic modulus of $3.3 \mathrm{TPa}$; the mass density of $2000 \mathrm{~kg} / \mathrm{m}^{3}$. The foundation modulus relative to the elastic medium, $k_{w}$, which can be obtained from the Whitney-Riley model, and is given as [42]:

$$
k_{w}=\frac{E_{m} L_{C N T}}{\left(1+\mu_{w}\right) r_{C N T}}
$$

where $E_{w}$ and $\mu_{w}$ are the elastic modulus and Poisson's ratio of the elastic medium, respectively. $r_{C N T}$ and $L_{C N T}$ are the radius of $C N T$ and the length of the clamped part, respectively. The elastic matrix 
was considered to be an epoxy resin, which had an elastic modulus of approximate $E_{m}=2.9 \mathrm{GPa}$ and Poisson's ratio of $\mu_{m}=0.25$.

CNTs have the resonant vibration frequency with different modes. Figure 2 plots three kinds of vibration mode shapes for CNTs under the axial compression load, in which the vibration frequency varies from low to high. The relationship between the vibration frequency and axial compressive load acting on CNT is shown in Figure 3 under the first and the second modes. The result shows that the vibration frequency of CNT decreased as axial load increased, tending to zero in a certain value called to the critical load, in which the vibration frequency decreased rapidly. In the simulation, the obtained critical load was $2.5 \mathrm{nN}$, which was smaller than the buckling load of $6 \mathrm{nN}$ according to our previous report [43]. This indicated that the CNT remained the stability of mechanical property due to being less than buckling load. Note that the value of the fundamental vibration frequency rapidly decreased when the compressive load acting on CNT was increased. The fundamental vibration frequency vanished when the axial compressive reached $2.5 \mathrm{nN}$. The critical load was about $23 \mathrm{nN}$ for the second vibration modes, and the vibration frequency became very sensitive to values of the critical load.

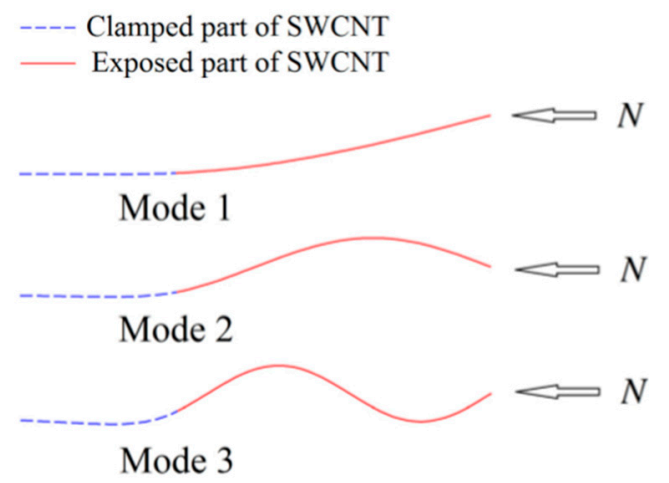

Figure 2. Schematic diagram of the first three vibration modes for AFM CNT tip with clamped elastic support.

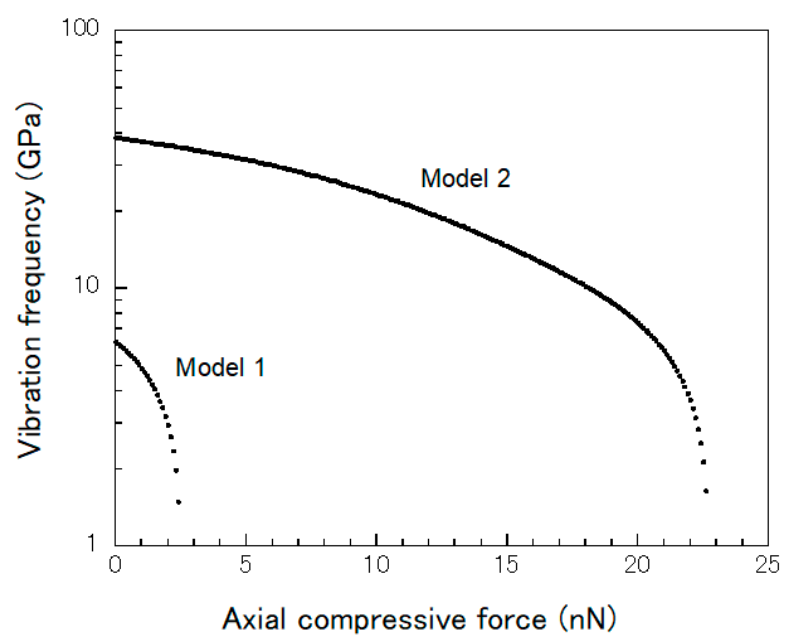

Figure 3. Relationship between the vibration frequency of AFM CNT tip and the axial compressive load for different vibration modes (CNT: Diameter $2 \mathrm{~nm}$, length $40 \mathrm{~nm}$, and lamped length $20 \mathrm{~nm}$ ).

In the following simulation and discussion, we mainly focused on the fundamental vibration mode. Figure 4 shows the relationship between the vibration frequency and the axial force acted on the CNT with different clamped length ratio of $L_{1} / L_{2}$. The effects of the length ratio on the vibration frequency of CNT were significant, especially for a smaller aspect ratio. The critical load decreased with decreasing clamping length of CNT, and the vibration frequency of CNT decreased largely due 
to the increasing instability in clamping part. Figure 5 shows the influences of clamping conditions, including the material constant of elastic medium and the clamping length on the vibration frequency of CNT under a load of $1 \mathrm{nN}$. It can be found that the vibration frequency of CNTs changed when the elastic modulus or the clamping length were smaller. The simulated result showed that the vibration frequency of CNT approached unity when the clamping length ratio reached certain values. The stiffness of the clamping medium was important for the measurement accuracy of the axial sensor, especially for low medium elastic modulus. In order to increase the measurement accuracy, the ratio of clamped to exposed lengths should be designed larger than 0.3 for an epoxy resin used as clamping materials, whose elastic modulus is about 3.0 GPa.

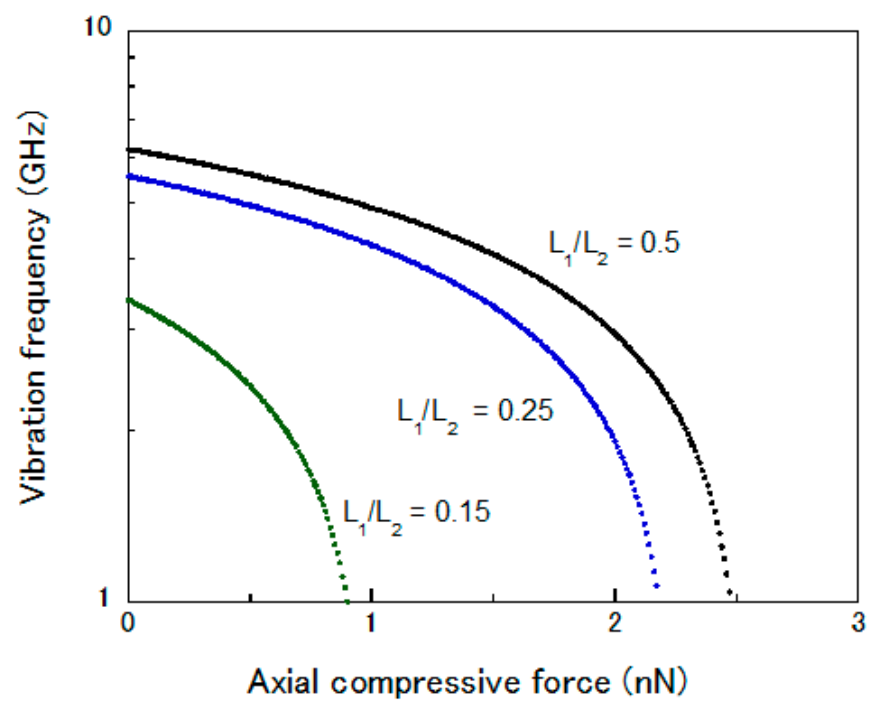

Figure 4. Variation of the vibration frequency of AFM CNT tip with the axial compressive load for different ratio of the clamped to exposed lengths (CNT: Diameter $2 \mathrm{~nm}$ and length $40 \mathrm{~nm}$ ).

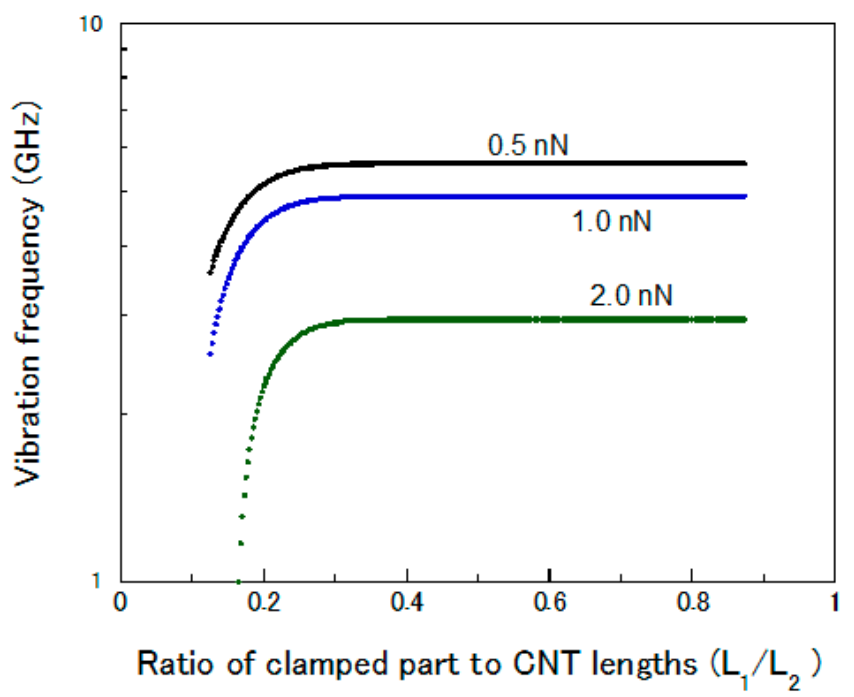

Figure 5. Variation of the vibration frequency of AFM CNT with the length ratio $L_{0} / L$ for different medium stiffness (CNT: Diameter $2 \mathrm{~nm}$ and length $40 \mathrm{~nm}$, and axial load $1 \mathrm{nN}$ ).

Figure 6 shows the influence of length-to-diameter ratio on the vibration frequency for CNT with a clamping length of $20 \mathrm{~nm}$. The CNT tips are subjected to the critical load of $0.5 \mathrm{nN}, 1.0 \mathrm{nN}$, and $2.0 \mathrm{nN}$, respectively. It is seen that the vibration frequency of CNTs decreased with increasing length-to-diameter ratio. The vibration frequency vanishes for a certain length-to-diameter under critical compressive loads. 


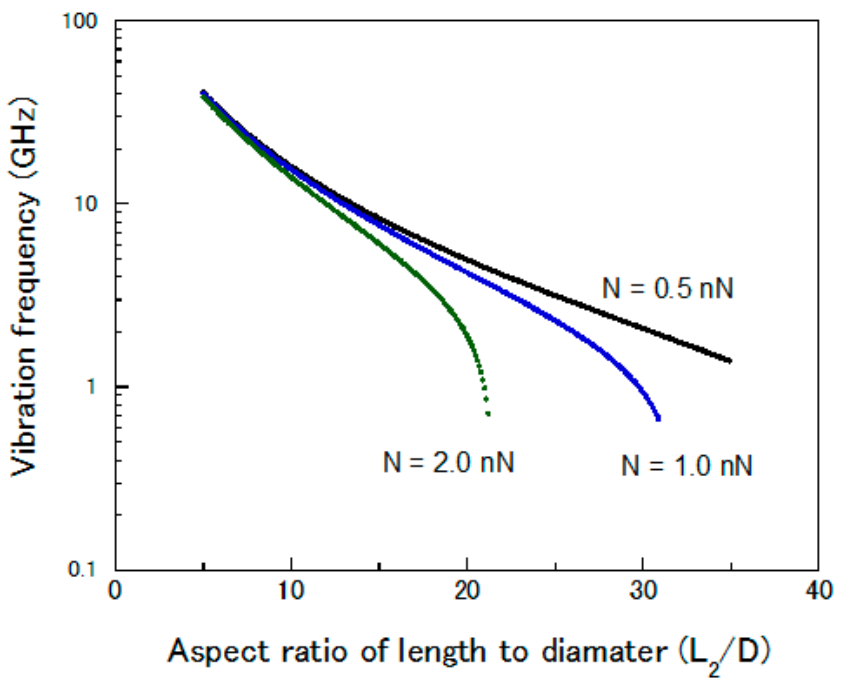

Figure 6. Variation of the vibration frequency of AFM CNT tip with the ratio of length to diameter under different the axial compressive load.

\section{Conclusions}

An analytical model and solution are employed to investigate the vibration frequency of CNT used as a nano-force sensor. The analytical model is considered as an AFM CNT tip, which has an elastic clamping part at one end, and is subjected to an axial compressive load at the exposed end. The clamping force between the elastic medium and CNTs can be determined by the Whitney-Riley model. The result suggests that the CNT can be effectively used as a nano-force sensor because the vibration frequency of a CNT is highly sensitive to an axial force load. The vibration frequency of the CNTs decreases with increasing compressive load. The simulation is very useful in design and analysis of CNTs used as a nano-force sensor.

Author Contributions: Conceptualization, and writing—original draft preparation, T.N.; formal analysis and program, K.U.

Funding: This research received no external funding.

Conflicts of Interest: The authors declare no conflict of interest.

\section{References}

1. Iijima, S. Helical microtubules of graphitic carbon. Nature 1991, 354, 56-58. [CrossRef]

2. Thostenson, E.T.; Renb, Z.; Chou, T.W. Advances in the science and technology of carbon nanotubes and their composites: A review. Compos. Sci. Technol. 2001, 61, 1899-1912. [CrossRef]

3. Natsuki, T.; Matsuyama, T. Vibration analysis of carbon nanotube-based resonator using nonlocal elasticity theory. Appl. Phys. A. 2015, 120, 1309-1313. [CrossRef]

4. Martinez, J.; Yuzvinsky, T.D.; Fennimore, A.M.; Zettl, A.; Garcia, R.; Bustamante, C. Length control and sharpening of atomic force microscope carbon nanotube tips assisted by an electron beam. Nanotechnology 2005, 16, 2493-2496. [CrossRef]

5. Druzhinina, T.S.; Hoeppener, S.; Schubert, U.S. Microwave assisted fabrication of carbon nanotube AFM Tips. Nano Lett. 2010, 10, 4009-4012. [CrossRef] [PubMed]

6. Irina, V.Z.; Natalia, P.B.; Yuri, N.P.; Lev, V.K. Carbon nanotubes: Sensor properties. A review. Mod. Electron. Mater. 2016, 2, 95-105.

7. Vera, S.; Suchol, S.; Maggie, H.; Sibo, L.; Timothy, M.S. Carbon nanotube chemical sensors. Chem. Rev. 2019, 119, 599-663.

8. Strus, M.C.; Raman, A.; Han, C.S.; Nguyen, C.V. Imaging artefacts in atomic force microscopy with carbon nanotube tips. Nanotechnology 2005, 16, 2482-2492. [CrossRef] 
9. Alizadegan, R.; Liao, A.D.; Xiong, F.; Pop, E.; Hsia, K.J. Effects of tip-nanotube interactions on atomic force microscopy imaging of carbon nanotubes. Nano Res. 2012, 5, 235-247. [CrossRef]

10. Hafner, J.H.; Cheung, C.L.; Oosterkamp, T.H.; Lieber, C.M. High-yield assembly of individual single-walled carbon nanotube tips for scanning probe microscopies. J. Phys. Chem. B 2001, 105, 743-746. [CrossRef]

11. Lamprecht, C.; Danzberger, J.; Lukanov, P.; Tilmaciu, C.M.; Galibert, A.M.; Soula, B.; Flahaut, E.; Gruber, H.J.; Hinterdorfer, P.; Ebner, A.; et al. AFM imaging of functionalized double-walled carbon nanotubes. Ultramicroscopy 2009, 109, 899-906. [CrossRef] [PubMed]

12. Wilson, N.R.; Macpherson, J.V. Carbon nanotube tips for atomic force microscopy. Nat. Nanotechnol. 2009, 4, 483-491. [CrossRef] [PubMed]

13. Wade, L.A.; Shapiro, I.R.; Ma, Z.; Quake, S.R.; Collier, C.P. Correlating AFM probe morphology to image resolution for single-wall carbon nanotube tips. Nano Lett. 2004, 4, 725-731. [CrossRef]

14. Nguyen, C.V.; Stevens, R.M.D.; Barber, J.; Han, J.; Meyyappan, M.; Sanchez, M.I.; Larson, C.; Hinsberg, W.D. Carbon nanotubes canning probe for profiling of deep-ultraviolet and $193 \mathrm{~nm}$ photoresist patterns. Appl. Phys. Lett. 2002, 81, 901-903. [CrossRef]

15. Chen, L.; Cheung, C.L.; Ashby, P.D.; Lieber, C.M. Single-walled carbon nanotube AFM probes: Optimal imaging resolution of nanoclusters and biomolecules in ambient and fluid environments. Nano Lett. 2004, 4, 1725-1731. [CrossRef]

16. Choi, N.; Uchihashi, T.; Nishijima, H.; Ishida, T.; Mizutani, W.; Akita, S.; Nakayama, Y.; Ishikawa, M.; Tokumoto, H. Atomic force microscopy of single-walled carbon nanotubes using carbon nanotube tip. Jpn. J. Appl. Phys. 2000, 39, 3707-3710. [CrossRef]

17. Yan, Y.; Miao, J.; Yang, Z.; Xiao, F.X.; Yang, H.B.; Liu, B.; Yang, Y. Carbon nanotube catalysts: Recent advances in synthesis, characterization and applications. Chem. Soc. Rev. 2015, 44, 3295-3346. [CrossRef] [PubMed]

18. Eveleens, C.A.; Irle, S.; Page, A.J. How does acetonitrile modulate single-walled carbon nanotube diameter during CVD growth? Carbon 2019, 146, 535-541. [CrossRef]

19. Hafner, J.H.; Cheung, C.L.; Lieber, C.M. Growth of nanotubes for probe microscopy tips. Nature 1999, 398, 761-762. [CrossRef]

20. Mori, H.; Ogata, S.; Li, J.; Akita, S.; Nakayama, Y. Plastic bending and shape-memory effect of double-wall carbon nanotubes. Phys. Rev. B 2017, 76, 165405. [CrossRef]

21. Somada, H.; Yoshikawa, Y.; Nagataki, A.; Hirahara, K.; Nakayama, Y. Energy barrier for disappearance of buckling to form a plastic bend in carbon nanotubes. Jpn. J. Appl. Phys. 2007, 46, 1055-1057. [CrossRef]

22. Natsuki, T.; Ni, Q.-Q.; Elishakoff, I. Influence of the axial compression on the natural frequency of AFM probes using double-walled carbon nanotubes with different wall lengths. Appl. Phys. A 2013, 110, 1-7. [CrossRef]

23. Natsuki, T. Theoretical analysis of vibration frequency of graphene sheet used as nanomechanical mass sensor. Electronics 2015, 4, 723-738. [CrossRef]

24. Yap, H.W.; Lakes, R.S.; Carpick, R.W. Mechanical instabilities of individual multiwalled carbon nanotubes under cyclic axial compression. Nano Lett. 2007, 7, 1149-1154. [CrossRef] [PubMed]

25. Kuzumaki, T.; Mitsuda, Y. Nanoscale mechanics of carbon nanotube evaluated by nanoprobe manipulation in transmission electron microscope. Jpn. J. Appl. Phys. 2006, 45, 364-368. [CrossRef]

26. Kutana, A.; Giapis, K.P. Transient deformation regime in bending of single-walled carbon nanotubes. Phys. Rev. Lett. 2006, 97, 245501. [CrossRef]

27. Farajpour, A.; Rastgoo, A. Influence of carbon nanotubes on the buckling of microtubule bundles in viscoelastic cytoplasm using nonlocal strain gradient theory. Results Phys. 2017, 7, 1367-1375. [CrossRef]

28. Cheung, C.L.; Hafner, J.H.; Lieber, C.M. Carbon nanotube atomic force microscopy tips: Direct growth by chemical vapor deposition and application to high-resolution imaging. Proc. Natl. Acad. Sci. USA 2000, 97, 3809-3813. [CrossRef]

29. Yakoboson, B.I.; Brabec, C.J.; Bernholc, J. Nanomechanics of carbon tubes: Instabilities beyond linear response. Phys. Rev. Lett. 1996, 76, 2511-2514. [CrossRef]

30. Wang, C.Y.; Zhang, Y.Y.; Wang, C.M.; Tan, V.B. Buckling of carbon nanotubes: A literature survey. J. Nanosci. Nanotechnol. 2007, 7, 4221-4247. [CrossRef]

31. Shima, H. Buckling of carbon nanotubes: A state of the art review. Materials 2012, 5, 47-84. [CrossRef] [PubMed] 
32. Garcia, R.; Herruzo, E.T. The emergence of multifrequency force microscopy. Nat. Nanotechnol. 2012, 49, 217-226. [CrossRef] [PubMed]

33. Barretta, R.; Luciano, R.; de Sciarra, F.M.; Ruta, G. Simulation of the thermal shock behavior of ultra-high temperature ceramics with the consideration of temperature-dependent crack propagation criterion and interaction between thermal shock cracks evolution and thermal conduction. Eur. J. Mech. A Solids 2018, 72, 268-274.

34. Apuzzo, A.; Barretta, R.; Faghidian, S.A.; Luciano, R.; de Sciarra, F.M. Free vibrations of elastic beams by modified nonlocal strain gradient theory. Int. J. Eng. Sci. 2018, 133, 99-108. [CrossRef]

35. Li, L.; Hu, Y.; Li, X. Longitudinal vibration of size-dependent rods via nonlocal strain gradient theory. Int. J. Mech. Sci. 2016, 115, 135-144. [CrossRef]

36. De Sciarra, F.M. A nonlocal model with strain-based damage. Int. J. Solids Struct. 2009, 46, 4107-4122. [CrossRef]

37. Barretta, R.; Fabbrocino, F.; Luciano, R.; de Sciarra, F.M. Closed-form solutions in stress-driven two-phase integral elasticity forbending of functionally graded nano-beams. Physica E 2018, 97, 13-30. [CrossRef]

38. De Sciarra, F.M. On non-local and non-homogeneous elastic continua. Int. J. Solids Struct. 2009, 46, 651-676. [CrossRef]

39. De Sciarra, F.M. Variational formulations, convergence and stability properties in nonlocal elastoplasticity. Int. J. Solids Struct. 2008, 45, 2322-2354. [CrossRef]

40. De Sciarra, F.M. Novel variational formulations for nonlocal plasticity. Int. J. Plast. 2009, 25, 302-331. [CrossRef]

41. Shi, J.X.; Natsuki, T.; Lei, X.W.; Ni, Q.-Q. Buckling instability of carbon nanotube atomic force microscope probe clamped in an elastic medium. J. Nanotechnol. Eng. Med. 2012, 3, 020903. [CrossRef]

42. Yang, H.K.; Wang, X. Torsional buckling of multi-wall carbon nanotubes embedded in an elastic medium. Compos. Struct. 2007, 77, 182-192. [CrossRef]

43. Natsuki, T.; Ni, Q.-Q.; Endo, M. Stability analysis of double-walled carbon nanotubes as AFM probes based on a continuum model. Carbon 2011, 49, 2532-2537. [CrossRef] 